\title{
A transfer matrix method for the analysis of fractal quantum potentials
}

\author{
Juan A. Monsoriu ${ }^{1 *}$, Francisco R. Villatoro ${ }^{2}$, María J. Marín $^{3}$, \\ Javier F. Urchueguía ${ }^{1}$ and Pedro Fernández de Córdoba ${ }^{4}$ \\ ${ }^{1}$ Departamento de Física Aplicada, Universidad Politécnica de Valencia, E-46022 \\ Valencia, Spain \\ 2 Departamento de Lenguajes y Ciencias de la Computación, Universidad de Málaga, \\ E-29071 Málaga, Spain \\ ${ }^{3}$ Departamento de Termodinámica, Universitat de València, E-46100 Burjassot, \\ Spain \\ ${ }^{4}$ Departamento de Matemática Aplicada, Universidad Politécnica de Valencia, \\ E-46022 Valencia, Spain
}

\begin{abstract}
The scattering properties of quantum particles on fractal potentials at different stages of fractal growth are obtained by means of the transfer matrix method. This approach can be easily adopted for project assignments in introductory quantum mechanics for undergraduates. The reflection coefficients for both the fractal potential and the finite periodic potential are calculated and compared. It is shown that the reflection coefficient for the fractal has a self-similar structure associated with the fractal distribution of the potential.
\end{abstract}

PACS numbers: 03.65.Nk, 05.45.Df

Submitted to: Eur. J. Phys.

\footnotetext{
* To whom correspondence should be addressed (jmonsori@fis.upv.es)
} 


\section{Introduction}

Both quantum mechanics and elementary solid state physics courses illustrate the energy band structure in solids through the one-dimensional Kronig-Penney model that consists of a periodic configuration of square-well potentials [1, 2. This problem is usually solved by matching the boundary conditions of the wavefunctions at the cell boundaries, thus requiring the computation of the determinant of a $4 \times 4$ matrix [3]. Recently, some less tedious approaches have been proposed which usually can be readily adapted to finite periodic potentials [4. Among these methods, those based on the transfer matrix approach which only uses $2 \times 2$ matrix operations in a purely algebraic way are the most appropriate ones for beginners [5. Moreover, this method allows to introduce a numerical method based on a piecewise constant approximation [6] for a general potential, the analysis of defects on slightly aperiodic potentials, and even the consideration of more complicated potentials. Among the last, fractal potentials is the one which we considered here.

In recent years the study of fractals has attracted much attention because many physical phenomena, natural structures and statistical processes can be analyzed and described by using a fractal approach 7, 8. From a mathematical point of view, fractals are self-similar structures obtained by performing a basic operation, called generator, on a given geometrical object called initiator, and repeating this process on multiple levels; in each one of them, an object composed of sub-units of itself is created that resembles the structure of the whole object. Mathematically, this property should hold in all scales. However, in the real world, there are lower and upper bounds over which such self-similar behavior applies. Fractals are becoming a useful tool to be able to model diverse physical systems [9, 10, and have new technological applications [11, 12].

In non-relativistic quantum mechanics, fractals have been used to generate new solutions of the Schrödinger equation which are continuous but nowhere differentiable wave functions 13, and models for the so-called fractal potentials 14. Fractal potentials allow the analysis of quasi-periodic and nearly stochastic potentials using the symmetries induced by the self-similar structure of the potential. Here we consider the simplest fractal, the (triadic) Cantor set, as a fractal potential for quantum scattering [15] and tunnelling [16, 17].

In this paper, we present a simple transfer matrix method to obtain the scattering properties of Cantor set fractal potentials, which can easily be automated by computers. The present method makes easier for the comparison with the finite periodic case and shows how the reflection coefficient for the fractal case has a self-similar structure associated with the fractal distribution of the potential. Moreoever, it can be easily implemented in any computer language, e.g., the Mathematica software package, accesible to undergraduate students with only a basic programming experience, so that it can be adopted for project assignments in computer physics courses. Starting with the implementation of the transfer matrix method for a potential barrier, the extension to finite periodic potential is a straightforward one; its extension to Cantor set potentials 


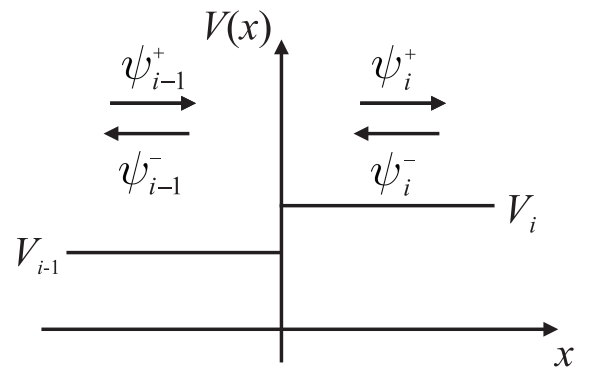

(a)

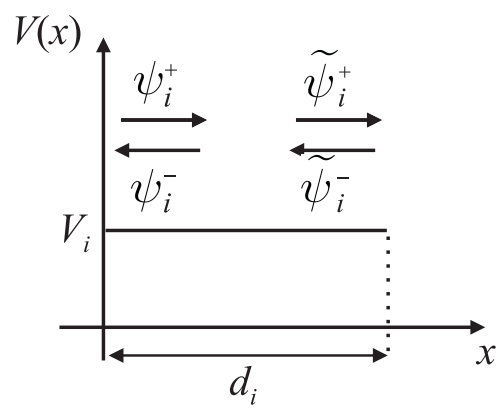

(b)

Figure 1. (a) Local scattering with the $i$-th interface of the piecewise constant potential among the values $V_{i-1}$ and $V_{i}$. (b) Wave propagation through the constant potential $V_{i}$ as used to calculate the propagation matrix.

can be based on a recursive implementation, involving the possible improvement of student programming skills. Furthermore, these projects can introduce the students to the analysis of computational complexity of algorithms, since the Cantor set prefractal has a large number of potential barriers and its simulation requires an exponential number of matrix products.

This paper is organized as follows: The next section describes the main facts about the transfer matrix method for quantum scattering implemented by piecewise constant potentials. In Section 3, the reflection coefficient for the tunnelling on both the finite periodic potential as well as the Cantor set pre-fractals are determined and compared. Finally, the last section is devoted to the conclusions.

\section{The transfer matrix method in quantum scattering}

Let us consider the one-dimensional, steady-state, linear Schrödinger equation

$$
-\frac{\hbar^{2}}{2 m} \frac{\partial^{2} \psi(x)}{\partial x^{2}}+V(x) \psi(x)=E \psi(x),
$$

where $\psi(x), m$ and $E$ are the wavefunction, mass and energy of the particle, respectively. The constant $\hbar$ is Planck's constant, and $V(x)$ is the quasiperiodic potential which can be represented by a piecewise constant function. Figure 1(a) shows the quantum scattering at the $i$-th interface between two successive constant values of the piecewise potential, whose position, without loss of generality, has been taken as $x=0$. In this figure, both $\psi_{i}^{+}$and $\psi_{i}^{-}$are forward and backward plane wavefunctions, respectively, on the region where the potential value is $V_{i}$, and $\psi_{i}=\psi_{i}^{+}+\psi_{i}^{-}$. These wave functions are given by

$$
\psi_{i}^{ \pm}=A_{i}^{ \pm} e^{ \pm \mathrm{i} k_{i} x}
$$

where $k_{i}=\frac{1}{\hbar} \sqrt{2 m\left(E-V_{i}\right)}$ is the local particle momentum, and $A_{i}^{ \pm}$are integration constants to be determined by applying the standard boundary conditions at the 


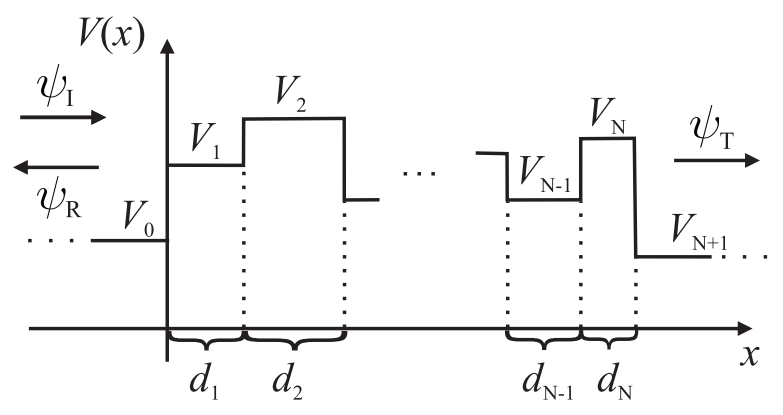

Figure 2. Piecewise constant potential with $N$ potential wells $V_{i}$ with $d_{i}$ as the corresponding width. $V_{0}$ and $V_{N+1}$ are the surrounding constant potential values extended to infinity.

interface. The continuity of the wavefunctions and the derivatives at the boundary are given by

$$
\begin{array}{ll}
\psi_{i-1}(x=0)=\psi_{i}(x=0), & A_{i-1}^{+}+A_{i-1}^{-}=A_{i}^{+}+A_{i}^{-}, \\
\psi_{i-1}^{\prime}(x=0)=\psi_{i}^{\prime}(x=0), & k_{i-1} A_{i-1}^{+}-k_{i-1} A_{i-1}^{-}=k_{i} A_{i}^{+}-k_{i} A_{i}^{-},
\end{array}
$$

where the prime denotes differentiation. Eq. (3) is a linear system of equations which can be written in matrix notation as

$$
\left(\begin{array}{cc}
1 & 1 \\
k_{i-1} & -k_{i-1}
\end{array}\right)\left(\begin{array}{c}
A_{i-1}^{+} \\
A_{i-1}^{-}
\end{array}\right)=\left(\begin{array}{cc}
1 & 1 \\
k_{i} & -k_{i}
\end{array}\right)\left(\begin{array}{c}
A_{i}^{+} \\
A_{i}^{-}
\end{array}\right),
$$

and yielding

$$
\left(\begin{array}{c}
A_{i-1}^{+} \\
A_{i-1}^{-}
\end{array}\right)=D_{i-1}^{-1} D_{i}\left(\begin{array}{c}
A_{i}^{+} \\
A_{i}^{-}
\end{array}\right), \quad D_{i}=\left(\begin{array}{cc}
1 & 1 \\
k_{i} & -k_{i}
\end{array}\right) .
$$

Here on, the matrix $D_{i-1}^{-1} D_{i}$ is referred to as the wave scattering matrix.

After crossing the $i$-th interface, the plane wave propagates through the constant potential $V_{i}$ until it finds the next interface at a distance $d_{i}$. Using the notation shown in Figure 1(b), this wavefunction is given by

$$
\widetilde{\psi}_{i}^{ \pm}=A_{i}^{ \pm} e^{ \pm \mathrm{i} k_{i} d_{i}} e^{ \pm \mathrm{i} k_{i} x}=\widetilde{A}_{i}^{ \pm} e^{ \pm \mathrm{i} k_{i} x},
$$

and a wave propagation matrix $P_{i}$ can be defined as

$$
\left(\begin{array}{c}
\widetilde{A}_{i-1}^{+} \\
\widetilde{A}_{i-1}^{-}
\end{array}\right)=\left(\begin{array}{cc}
e^{\mathrm{i} k_{i} d_{i}} & 0 \\
0 & e^{-\mathrm{i} k_{i} d_{i}}
\end{array}\right)\left(\begin{array}{c}
A_{i}^{+} \\
A_{i}^{-}
\end{array}\right)=P_{i}\left(\begin{array}{c}
A_{i}^{+} \\
A_{i}^{-}
\end{array}\right) .
$$

Both the scattering and propagation matrices can be used to solve the general problem of the scattering with a piecewise constant potential with $N$ potential wells, as shown in Figure 2. The successive application of the scattering and propagation matrices yield

$$
\left(\begin{array}{c}
A_{0}^{+} \\
A_{0}^{-}
\end{array}\right)=D_{0}^{-1} D_{1}\left(\begin{array}{c}
A_{1}^{+} \\
A_{1}^{-}
\end{array}\right)=D_{0}^{-1} D_{1} P_{1} D_{1}^{-1} D_{2}\left(\begin{array}{c}
A_{2}^{+} \\
A_{2}^{-}
\end{array}\right),
$$




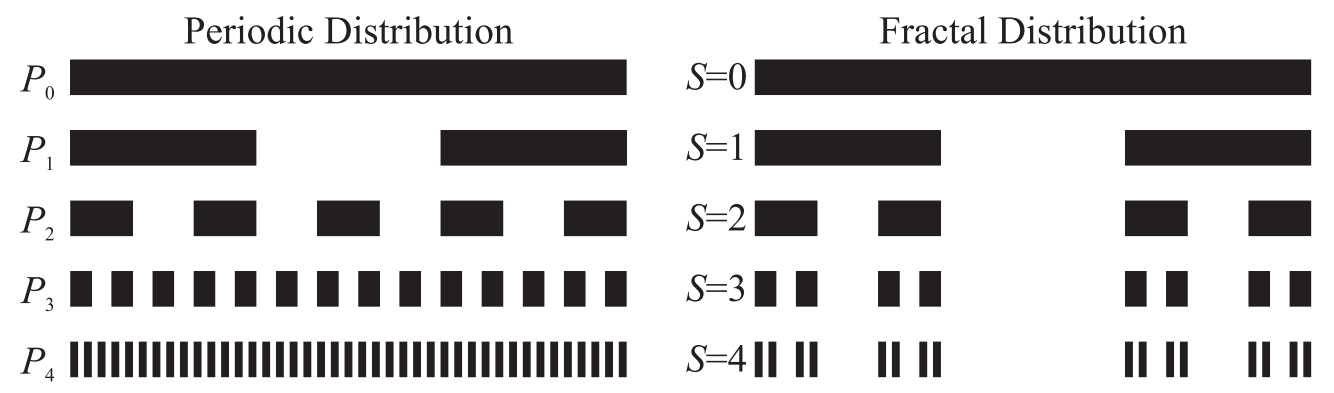

(a)

(b)

Figure 3. Finite periodic (a) and Cantor set quasiperiodic (b) potentials where the white and black regions denote the potential values 0 and $\mathcal{V}$, respectively.

and, in the most general form,

$$
\left(\begin{array}{c}
A_{0}^{+} \\
A_{0}^{-}
\end{array}\right)=M\left(\begin{array}{c}
A_{N+1}^{+} \\
A_{N+1}^{-}
\end{array}\right), \quad M=D_{0}^{-1}\left(\prod_{i=1}^{N} D_{i} P_{i} D_{i}^{-1}\right) D_{N+1} .
$$

Both the reflection and transmission coefficients of the scattering of a quantum particle, incoming from the left, with the $N$-well potential is determined by the coefficients of the matrix $M$,

$$
\left(\begin{array}{c}
A_{0}^{+} \\
A_{0}^{-}
\end{array}\right)=\left(\begin{array}{ll}
M_{11} & M_{12} \\
M_{21} & M_{22}
\end{array}\right)\left(\begin{array}{c}
A_{N+1}^{+} \\
0
\end{array}\right)
$$

where no backward moving particle can be found on the right side of the potential, so $A_{N+1}^{-}=0$. The reflection and the transmission coefficients [2, 18] are given by

$$
R=\frac{\left|A_{0}^{-}\right|^{2}}{\left|A_{0}^{+}\right|^{2}}=\frac{\left|M_{21}\right|^{2}}{\left|M_{11}\right|^{2}}, \quad \text { and } \quad T=\frac{k_{N+1}\left|A_{N+1}^{+}\right|^{2}}{k_{0}\left|A_{0}^{+}\right|^{2}}=\frac{k_{N+1}}{k_{0}\left|M_{11}\right|^{2}}
$$

respectively.

\section{Presentation of results}

The simplest fractal potential is the Cantor set, shown in Figure 3(b), which can be obtained by means of an iterative construction. The first step $(S=0)$ is to take a segment of unit length. The next one $(S=1)$ is to divide the segment in three equal parts of length $1 / 3$ and remove the central one. In general, at the stage $S$, there are $2^{S}$ segments of length $3^{-S}$ with $2^{S}-1$ gaps in between. Stage $S+1$ is obtained by dividing each of these segments into three parts of length $3^{-S-1}$ and removing the central ones. In Figure 3(b), only the four first stages are shown for clarity. Note that the $S$-th stage Cantor set pre-fractal can be interpreted as a quasiperiodic distribution of segments

which can be obtained by removing some segments in a finite periodic distribution as shown in Figure 3(a). This distribution at stage $p_{M}$ has $\left(3^{M}-1\right) / 2+1$ potential barriers 
of length $3^{-M}$, separated by potential wells of the same length, so the "period" of this finite structure is $\Lambda=2 \cdot 3^{-M}$.

The scattering problem for both the quasiperiodic, Cantor set, pre-fractal potential, and the finite periodic potential can be easily solved by means of the matrix transfer theory presented in Sec. 2. It is standard to normalize both the energy and the height of the potential barrier by the period $\Lambda$, introducing the non-dimensional variables

$$
\phi=\Lambda \frac{\sqrt{2 m E}}{\hbar}, \quad \text { and } \quad \phi_{\mathcal{V}}=\Lambda \frac{\sqrt{2 m \mathcal{V}}}{\hbar} .
$$

Figures 4 and 5 show the reflection coefficient, $R$, for the finite periodic potential and Cantor set fractal potential, respectively, around the interval which contains the first band gap of the infinite periodic one. Using the standard Kronig-Penney model [19], this band gap can be numerically calculated yielding $3.2519<\phi<3.6222$ for the potential $\phi_{\mathcal{V}}=2$. In this energy interval, a Bloch wavefunction does not propagate in a infinite periodic potential and, therefore, the transmission coefficient should vanish $(R=1)$. Only evanescent wavefunctions characterized by a complex wavevector, $k$, are solutions of the Schrödinger equation. For this reason, when the number of periods is finite, the quantum particle may pass through the potential distribution by the tunnelling effect. Figure 4 shows that the reflection coefficient approaches unity as the number of periods in the spatial interval increases, illustrating the process of appearance of the band gap of the (full) periodic structure. Although, at the graphical resolution of Figure प(c) the value $R=1$ is apparently reached, the reflection coeficient is always smoller than unity in finity peridodic structures.

Figure 5 shows the reflection coefficient for the Cantor set pre-fractal potential for $S=2$ (top), $S=3$ (middle) and $S=4$ (bottom). It is shown that the reflection at each higher stage is a modulated version of that associated with the previous stage. That is, the reflection spectrum exhibits a characteristic fractal profile that reproduces the selfsimilarity of the potential distribution. In fact, any wide peak at stage $S$ is transformed into three narrower and taller peaks at stage $S+1$. Zero reflection from these fractal quantum potential occurs at specific discrete energies, while near total reflection is possible at other discrete energies. Comparing Figures 4 and 5, an increasing number of zeros inside the band gap is observed. These zeros represent resonances due to the presence of "defects" in the quasiperiodic potential obtained by removing some segments in the finite periodic sequence.

\section{Conclusions}

The transfer matrix method is becoming the standard method for the calculation of the tunnelling of quantum particles on constant piecewise potentials because it can be used for simple, textbook-like problems and as a numerical method for computer simulations. This procedure has been applied to Cantor set fractal potentials, which are constant value potentials with support on a Cantor set. For pre-fractals, the $S$-th stage fractal, the reflection coefficient was numerically calculated and compared with that of 

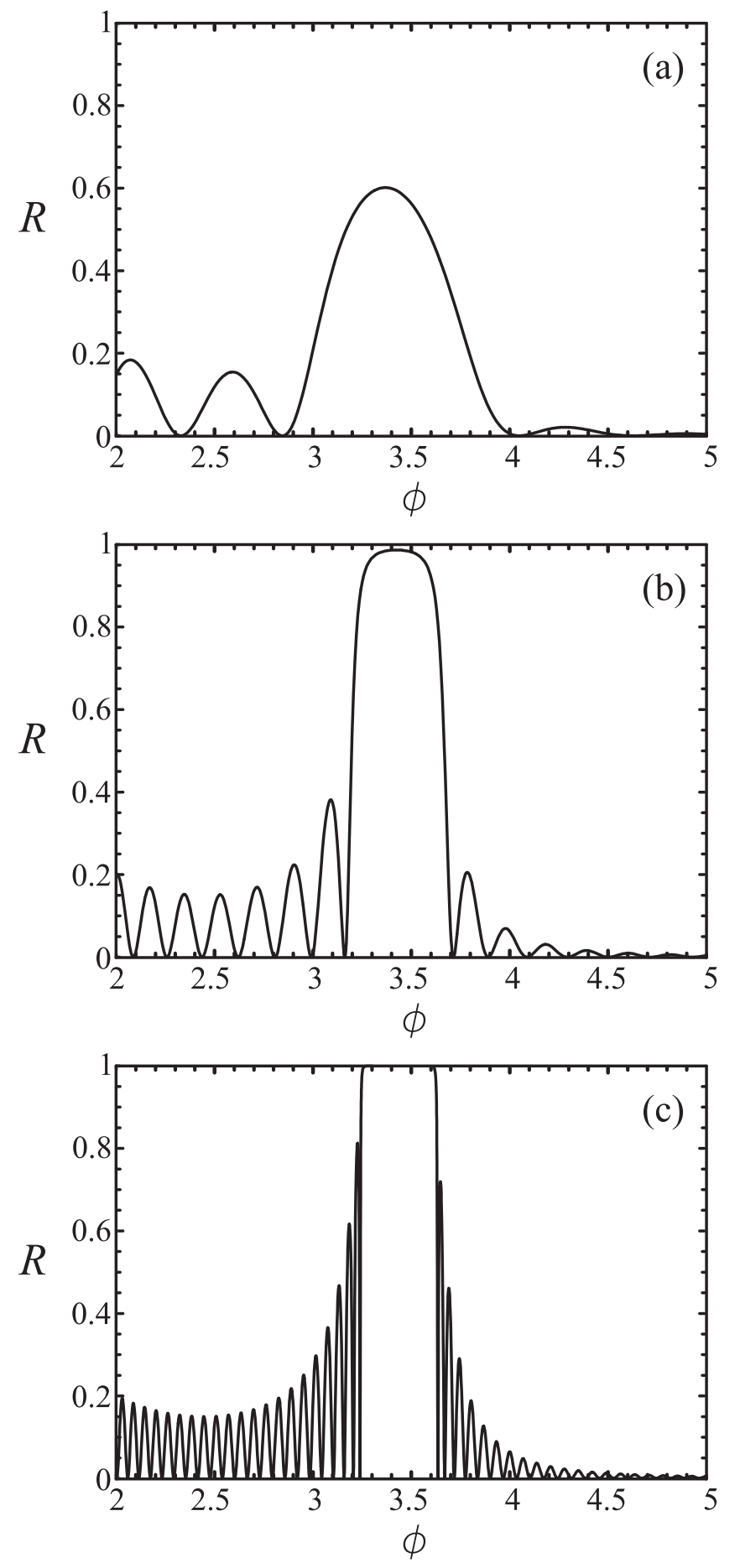

Figure 4. Scattering reflection coefficient for the finite periodic potentials of stages $p_{2}$ (a), $p_{3}$ (b), and $p_{4}$ (c) as a function of the normalized energy $\phi$ for the potential $\phi_{\mathcal{V}}=2$. 

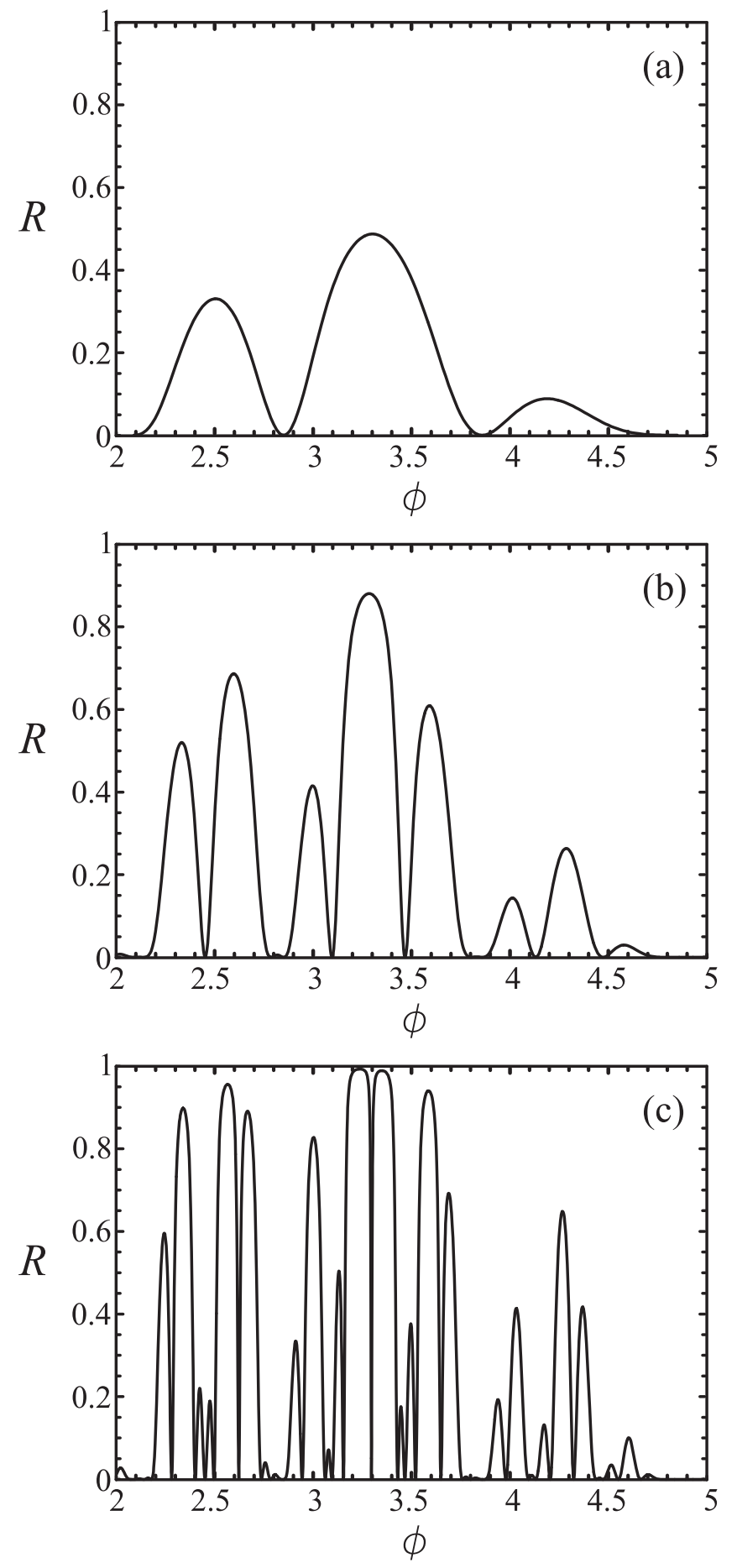

Figure 5. Scattering reflection coefficient for the Cantor set pre-fractal potentials of stages $S=2(\mathrm{a}), S=3(\mathrm{~b})$, and $S=4$ (c) as a function of the normalized energy $\phi$ for the potential $\phi_{\mathcal{V}}=2$. 
a finite periodic potential of the same period. The appearance of the first band gap of the Kronig-Penney model in the finite periodic potential has been illustrated. The reflection coefficient for the Cantor set potential is self-similar.

The transfer matrix method presented in this paper can be easily adopted in computer laboratories for undergraduate quantum mechanics courses, providing a powerful method for developing students skill on physics by means of computational tools. Furthermore, fractal geometry is a highly motivating topic for the students providing a great opportunity to undertake projects closely related to research ones.

\section{Acknowledgments}

The authors are thankful to Prof. Sarira Sahu from the Instituto de Ciencias Nucleares at the Universidad Autónoma de México, México, and Prof. Juan I. Ramos from the Universidad de Málaga, Spain, for their valuable comments and suggestions. J.A. Monsoriu and P. Fernández de Córdoba were supported by the Plan Nacional I+D+I under project TIC 2002-04527-C02-02 (Spain). F.R. Villatoro was supported by Project BFM2001-1902 from the Dirección General de Investigación, Ministerio de Ciencia y Tecnología, Spain. Part of this work was done during the visit of J.A. Monsoriu to the Universidad de Málaga with a grant from the Universidad Politécnica de Valencia, under the "Programa de Incentivo a la Investigación de la UPV 2004".

\section{References}

[1] Kittel C 1996 Introduction to Solid State Physics (Wiley, New York)

[2] Liboff R 2003 Introductory Quantum Mechanics (Benjamin Cummings, Redwood City, CA)

[3] Szmulowicz F 1997 Eur. J. Phys. 18392

[4] Sprung D W L, Sigetich J D, Wu H, and Martorell J 2000 Am. J. Phys. 68715

[5] Griffiths D J and Steinke C A 2001 Am. J. Phys. 69137

[6] Kalotas T M and Lee A R 1991 Eur. J. Phys. 12275

[7] Mandelbrot B B 1982 The Fractal Geometry of Nature (Freeman, San Francisco)

[8] Ficker T and Benesovsky P 2002 Eur. J. Phys. 23403

[9] Berry M and Klein S 1996 J. Mod. Opt. 432139

[10] Karman G P, McDonald G S, New G H C, and Woederman J P 1999 Nature 402138

[11] Saavedra G, Furlan W D, and Monsoriu J A 2003 Opt. Lett. 28971

[12] Monsoriu J A, Furlan W D, and Saavedra G 2004 Opt. Express 124227

[13] Wojcik D, Bialynicki-Birula I, and Zyczkowski K 2000 Phys. Rev. Lett. 855022

[14] Albeverio S and Koshmanenko V 2000 Rep. Math. Phys. 45307

[15] Makarov K A 1994 J. Math. Phys. 351522

[16] Chuprikov N L 2000 J. Phys. A: Math. Gen. 334293

[17] Chuprikov N L and Zhabin D N 2000 J. Phys. A: Math. Gen. 334309

[18] Fernández de Córdoba P and Urchueguía J 2004 Fundamentos de Física Cuántica para Ingeniería (Textbook, Servicio de Publicaciones, Universidad Politécnica de Valencia, Spain)

[19] Schulkin B, Sztancsik L, and Federici J F 2004 Am. J. Phys. 721051 